\title{
Analysis and Evaluation of Boundary Conditions for Direct Surface Structuring by Multi-Beam Interference
}

\author{
Michael Steger ${ }^{1}$ and Arnold Gillner ${ }^{1,2}$ \\ ${ }^{1}$ Chair for Laser Technology, RWTH Aachen University, Steinbachstr. 15, \\ 52074 Aachen, Germany \\ ${ }^{2}$ Fraunhofer Institute for Lasertechnology, Steinbachstr. 15, 52074 Aachen, Germany \\ E-Mail: michael.steger@ilt.fraunhofer.de
}

\begin{abstract}
Multi-Beam-Interference (MBI) is a promising approach for a more cost-efficient functionalization of surfaces by nano structuring. It allows the simultaneous structuring of a whole surface area in one process step. This area is directly laser ablated with the modulated intensity created by the interference of two or more coherent beams. In this study, we first analyze the different possibilities for MBI setups with regards to the number and the alignment of utilized beams used for direct surface structuring. The key factors for direct surface structuring are stability and contrast of the interference pattern. From these criteria 2-Beam interference is derived as optimal setup for the direct structuring of surfaces. In the second part of this work experiments with a ns- and a ps-laser are carried out and compared to the theory. For the ns-laser minimal structures sizes in the $100 \mathrm{~nm}$ regime on polyimide are feasible, for the ps-laser minimal periodicity of $400 \mathrm{~nm}$ on stainless steel. The measured interference contrast decreases significantly for shorter pulses.
\end{abstract}

DOI: $10.2961 /$ jlmn.2016.03.0003

Keywords: Multi-Beam interference, MBI, Nano structuring, Interference contrast, Surface texturing

\section{Introduction}

Micro and nano structuring and thus functionalization of surfaces are a key feature for many applications. The wide variety of functions for these applications ranges from wettability and tribology to optical properties [1-3]. The challenge in generating the desired surface functionality is not only the feasibility of the structuring process itself, but also the costs of this process. Most commonly, methods relying on lithographic processes are used which allow the reproduction of complex 3D structures. But lithography is very cost-intensive due to many complex process steps and limited material selection.

Multi-Beam Interference (MBI) is a promising approach for a more cost-efficient functionalization of surfaces. It allows the simultaneous nano to micro structuring of whole surface areas in one process step by direct laser ablation. The size of the simultaneously structured surface area is scalable with the maximal pulse energy of the laser source and thousands of structure elements can be generated with only one pulse. The patterns created by MBI are always periodic and can be modified by the number of beams and their alignment. The restriction to periodic patterns fits well to the usually also periodic patterns demanded for surface functionalization. Additionally MBI is not limited to structuring of plane surfaces but can be used to create hierarchical structures [4]. With MBI a nanostructure can easily be added to a preexisting microstructure. Also laser ablation, especially for ultrashort pulse (USP) laser, is relatively material independent and therefore allows the processing of a wide range of materials.

Currently MBI is mainly used for lithographic purposes as a flexible substitution of the cost intensive lithographic masks or to generate complex 3D structures in photoresist. While theoretical calculation and optimization of the interference patterns for different beam setups exist in literature, they rarely consider the specific constraints for direct surface structuring or compare their application in regard to direct surface texturing [5-7].

From an experimental point of view successful direct surface structuring with multi-beam interference on a wide range of materials is reported. For example the structuring of plastics as polyimide and peek [3,6,7,8,9,10], semiconductors as silicon [8] or various metals as steel, chrome or aluminum [11-13].As interference setups mainly 2 to 4 beams are used which allow the creation of wide range of different surface structures. The influence of the parameters as polarization or amplitude on the structuring process is investigated [14], but not the interference contrast.

The aim of this paper is to fill this gap by a theoretical analysis of MBI as a method for direct surface structuring, followed by a comparison of theoretically expected with experimentally achieved results. An important part of this investigation is the possibility to measure the effective interference contrast which is used in the experiments.

\section{Theoretical analysis of $\mathrm{MBI}$}

The interference pattern from MBI is created in the intersection volume of two or more coherent laser beams. The pattern is periodic and its periodicity $\Lambda$ depends on the incidence angle $\alpha$ of the intercepting beams and the laser wavelength $\lambda$. The interference pattern for a two-beam interference is shown in figure 1 . The periodicity of the pattern is $\Lambda=\lambda /(2 \sin \alpha)$ in the $\mathrm{x}$-axis while it is constant in the other axes. The 2-beam interference is the basic module of every 


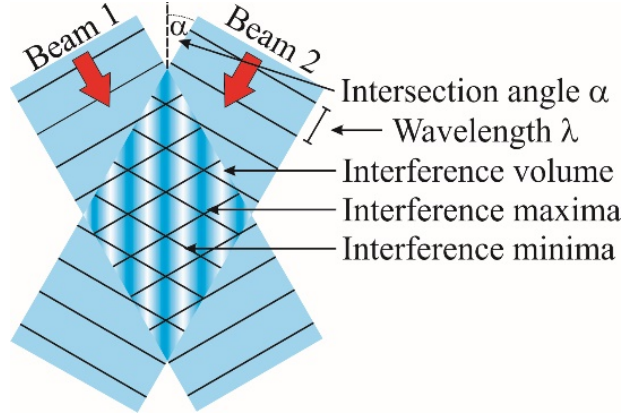

Fig. 1 Schematic diagram of a two-beam interference with wavelength $\lambda$ and an angle of incidence $\alpha$.

interference pattern. Every n-beam interference can be described as a sum of “ $n(n-1) / 2$ ” superposed 2-beam interferences.

The total time independent intensity distribution $I(\boldsymbol{r})$ in the interference volume the of $\mathrm{j}$ beams described by plane waves is given by [13]:

$$
\begin{gathered}
I(\boldsymbol{r})=\sum_{j} I_{j}(\boldsymbol{r})+2 \sum_{i<j} \sqrt{I_{i}(\boldsymbol{r}) I_{j}(\boldsymbol{r})} \cos \left(\theta_{i j}\right) \\
* \cos \left(\left(\mathbf{K}_{i}-\boldsymbol{K}_{j}\right) \boldsymbol{r}+\phi_{i}-\phi_{j}\right)
\end{gathered}
$$

Where $I_{j}$ is the intensity of beam $\mathrm{j}, \theta_{i j}$ is the angle between the unit vectors of beam $i$ and $j$ in its polarization direction, $\boldsymbol{K}_{j}$ is the wave vector and $\phi_{i}$ the phase of beam j.

The first sum of equation 1 describes the total intensity $I_{0}$ without interference. The second sum defines the modulation around $I_{0}$ due to the interference and is therefore referred to as interference term. Each summand of the term has the shape of a two beam interference. Depending on the ratio of the intensity $I_{j}$ of each wave and the angle $\theta_{i j}$ between them, the modulation depth ranges from 0 to $I_{0}$. This modulation depth is the interference contrast which is defined as:

$$
\text { Contrast }=\frac{I_{\max }-I_{\min }}{I_{\max }+I_{\min }}
$$

By means of multi-beam interference complex 3D-patterns can be generated. The first issue to be discussed for MBI as a method for direct nano structuring is which interference pattern is optimal to create surface features by laser ablation. In order to define the interference pattern, a set of criteria is necessary. Important criteria are:

- $\quad$ Periodicity of the pattern

- Form of the pattern

- $\quad$ Phase stability of the pattern

- $\quad$ Contrast of the pattern

The first two criteria focus on what kind of structures and in which sizes they can be generated by MBI. The periodicity of a pattern is strongly tied to the generated structure size. As it is especially interesting to produce sub- $\mu \mathrm{m}$ features with MBI, the minimal achievable size and the shape of the structures are important criteria. The shape of a pattern is primarily defined by the number of beams utilized. The second set of criteria corresponds to the stability and the quality of the interference modulation. Their influence on the ablation result is analyzed in the next section.

\subsection{Phase stability of the interference pattern}

The ablation of a surface is generated by the modulated intensity in the intersection plane between interference volume and sample surface. Therefore the stability of the intensity modulation in this intersection plane is important for the ablation results.

A phase shift in one of the beams involved in the creation of the interference pattern leads to a modification of the interference pattern. This modification can result in two kinds of changes of the interference pattern. In the first case a translation of the whole interference pattern occurs while in the second case the pattern itself changes. The outcome depends on the summation of interference terms. For more than three beams the pattern commonly changes, due to the fact that not all summands of the interference term contain the beam that shifted its phase and therefore only parts of pattern are shifted, while other parts do not vary.

Due to the translation of the pattern even in the first case the intensity modulation in the intersection plane can change for certain interference patterns as shown in figure 2.

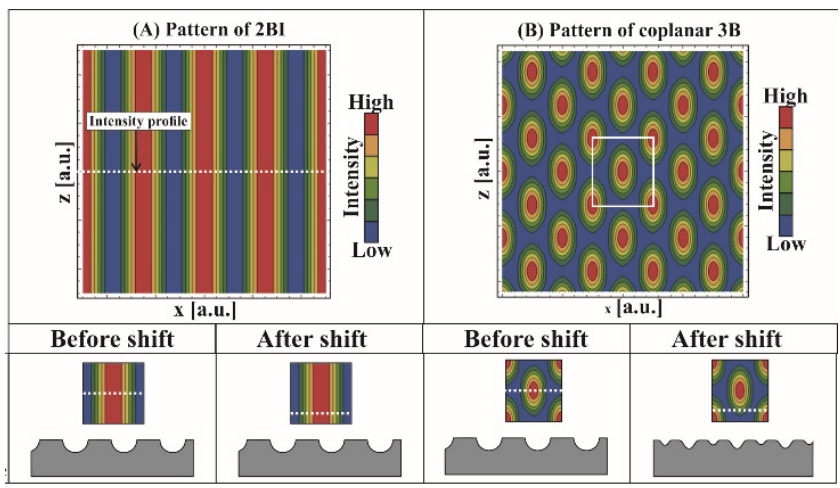

Fig. 2 Schematic diagram of the change of the generated structures for 2-beam and coplanar 3-beam interference in case of phase shift. In the upper row the intensity modulation perpendicular to the sample surface is shown for both interference patterns. The dotted line shows a possible intersection line of sample surface with the interference pattern. In the lower row ablation results for different intersection planes are presented. In (A) the intensity modulation in the intersection line between surface und interference pattern is conserved, while it changes for (B) and a different structure is generated on the surface.

If the intensity modulation in the intersection plane is altered, the ablated structure on the surface is also modified. It is important that the intensity modulation in the intersection plane is constant (apart from translation) in order to achieve stable structuring results due to the following reasons:

- Homogeneous structuring independent from the phase of each beam

- Initial phase relation of the beams is irrelevant

- Uneven surfaces (e.g. surface roughness or pre-structured surfaces) are structured with the same pattern

- Maximal structure depth is not limited by the spatial shape of interference pattern

This is not only important for multi-pulse structuring process but also relevant for single pulse ablation. If no phase stability is present, the initial state of phase relations are difficult to measure and additional optical components need to 
be integrated in the setup to control the phase. Without phase stability shifts in the optical path length below the laser wavelength can alter the generated pattern significantly.

\subsection{Contrast of the interference modulation}

The interference contrast describes the quality of the redistribution of the intensity due to interference. A gain in contrast means that the difference between the intensity maxima and minima of the interference modulation increases. Therefore the contrast is closely tied to the quality of the generated structures.

For a material with an ablation threshold of $F_{\text {thres }}$ the minimal intensity $I_{\text {min }}$, which corresponds to $F_{\text {min }}$, can be lower or higher than this threshold. If the minimal fluence is lower, ablation occurs only around the maximal fluence of the interference pattern. If the minimal fluence is higher than the ablation threshold, ablation occurs over the whole irradiated sample area and is only modulated in depth by the modulated intensity. An example for these two ablation regimes is shown in figure 3 where $d_{1}$ labels the depth of the structures. A simulation of the structure depth $d_{1}$ for a constant $F_{\text {ink }}$ of interference with varying contrast is shown in figure 4. $F_{\text {ink }}$ is the fluence without interference and the baseline around the modulation of interference occurs.

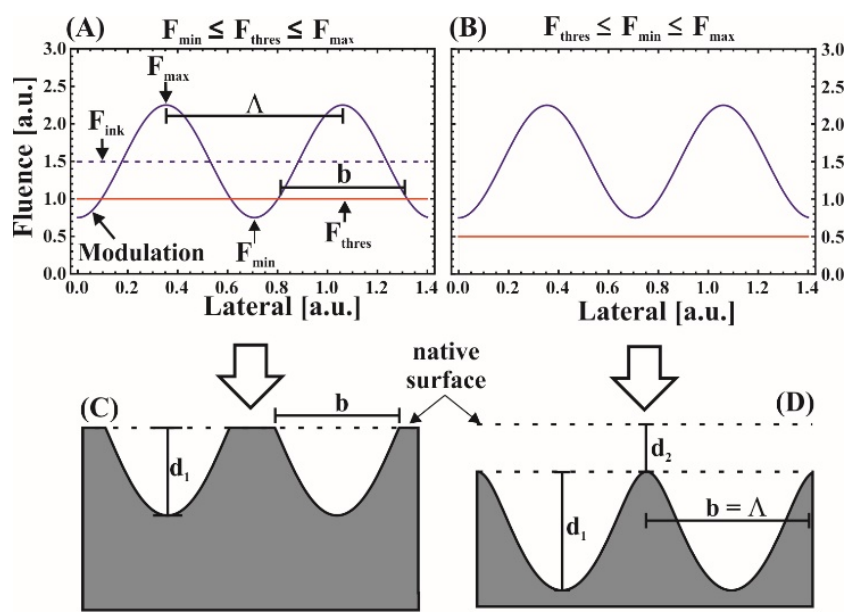

Fig. 3 Ablation results for different relations between the ablation threshold and fluence minima of the interference pattern. For (A) the fluence minima is below the ablation threshold which leads to a selective ablation around the maxima (C), while in (B) the intensity minima is above the threshold and ablation occurs over the whole sample surface (D).

In this simulation an ablation process based solely on the optical penetration depth is assumed [15]:

$$
d(\vec{r})=\frac{1}{\alpha} \log \left(\frac{F(\vec{r})}{F_{\text {thres }}}\right)
$$

A similar model is used in [3]. The depth of structures increases with the contrast of the interference (see figure 4 top). The ratio of the total unmodulated intensity $F_{\text {ink }}$ and the ablation threshold $F_{\text {thres }}$ defines two regimes with a ratio of 1 as borderline. For a ratio that is smaller than 1 ablation only occurs when the contrast is high enough so that the interference modulation surpasses the ablation threshold. For a ratio larger than 1 the depth of structures increases with a discontinuity in case the minimal fluence sinks below the ablation threshold. An interesting observation is that for this ablation model the structure depth is identical for any ratio of intensity vs. threshold before the discontinuity. Only the depth $d_{2}$ increases relative to the native surface (compare figure 3 ).

The width of structures shows a similar behavior and is divided into two regimes (see figure 4 bottom). For a ratio smaller than 1 the width of structures increases with the contrast toward a value of half of the periodicity of the pattern as soon as the ablation threshold is surpassed. For a ratio larger than 1 and for a low contrast the width equals the periodicity of the pattern due to the fact that ablation occurs over the whole surface. For a higher contrast the minimal fluence sinks below the threshold and the width decreases towards a value of half of the periodicity of the pattern.

A high contrast increases the aspect ratio of structures and leads to a more defined structure. Also the efficiency of the process is increased, because a certain structure depth can be achieved with less energy compared to a lower contrast. Therefore the energy deposited in an area in which no material alteration is desired is reduced and unwanted heating of the surface is avoided.
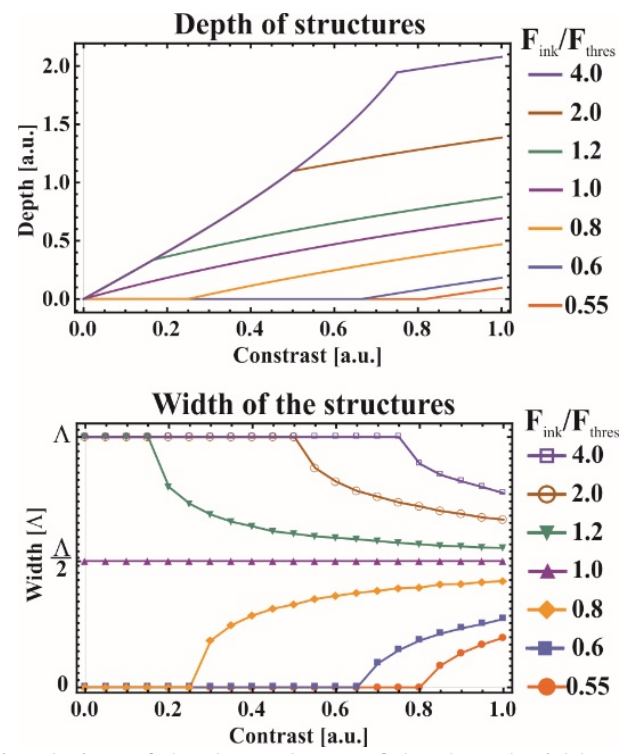

Fig. 4 Simulation of the dependence of depth and width against the contrast of an interference pattern for a constant total energy. $\mathrm{F}_{\text {ink }} / \mathrm{F}_{\text {thres }}$ is the ratio between the unmodulated fluence without interference and the ablation threshold of a material.

\subsection{Comparison of different interference patterns}

In table 1 an overview of the characteristics of different interference setups is presented. It is divided in symmetrical and asymmetrical setups. For a symmetrical setup all beams have the same angle to the surface normal and an even spacing to each other. A 2-beam interference yields the smallest periodicity compared to setups with more beams. For example for symmetrical 3- and 4-beams setups, the periodicity for a fixed intersection angle and wavelength increases by $15 \%$ and $40 \%$ respectively. If more than 3 beams are used in a symmetrical arrangement or more than 2 beams are used in a coplanar arrangement, the interference pattern loses its phase stability. The contrast of a pattern is only optimal for a 2-beam interference or other completely coplanar arrangements and it is also independent from the intersection angle. For other setups not all polarization unit vectors of the beams 
can be simultaneously parallel and therefore the contrast is reduced. If further constraints regarding the homogeneity of the interference pattern are applied the contrast is even further reduced. The complexity of a pattern is closely tied to the number of beams and the resulting number of interference terms. By visualizing the superposition of a 1D cosine modulation of 2-beams interference it can be easily concluded that 2 beams produce a 1D pattern, 3-beams a 2D pattern and $n>3$ beams a 3D pattern. For surface texturing only up to 2D patterns are useful due to strong absorption near the sample surface.

In conclusion 2-beams interference is the ideal setup for direct surface structuring under the here presented criteria apart from its limitation to a 1D pattern. This limitation can be circumvented by two structuring steps when, in between the two steps, the surface or the pattern is rotated.

Table 1 Comparison of the interference patterns

\begin{tabular}{|c|c|c|c|c|c|}
\cline { 2 - 6 } \multicolumn{1}{c|}{} & Alignment & $\begin{array}{c}\text { Phase } \\
\text { Stability }\end{array}$ & Contrast & Periodicity & $\begin{array}{c}\text { Possible } \\
\text { pattern }\end{array}$ \\
\hline 2 beams & Symmetrical & $\checkmark$ & Optimal & $\Lambda_{2 B}=\frac{\lambda}{2 \sin (\alpha)}$ & 1D \\
\hline 3 beams & Symmetrical & $\checkmark$ & Reduced & $\Lambda_{3 B}=2 / \sqrt{3} \Lambda_{2 B}$ & 2D \\
\hline 4 beams & Symmetrical & $X$ & Reduced & $\Lambda_{4 B}=\sqrt{2} \Lambda_{2 B}$ & $3 D$ \\
\hline \multirow{2}{*}{ N Beams } & Symmetrical & $X$ & Reduced & Increasing with N & $3 D$ \\
\cline { 2 - 6 } & Asymmetrical & $X$ & Reduced & Increasing with N & 3D \\
\hline
\end{tabular}

\section{Experimental setup}

As shown in figure 5 the beam from a laser source is slightly focused by a lens before it is split into two parts by a diffractive optical element. Apart from the -1 and the 1order all other beams are blocked by a filter. The two remaining identical beams are guided through a prism and two mirrors to intersect at the sample surface. The polarization of both beams is parallel to each other und perpendicular to the plane of incidence. To adjust the optical path length of both beams relative to each other, a translation stage is integrated in one beam path. As beam sources two different laser are used: A diode pumped Nd:YAG laser (Q301-HD, JDSU) which operates at a wavelength of $\lambda=355 \mathrm{~nm}$ with a pulse duration of $\tau=38 \mathrm{~ns}$. The coherence length is $5.2 \mathrm{~mm}$. The second laser is a diode pumped $\mathrm{Nd}: \mathrm{YVO}_{4}$ MOPA laser (Master Oscillator Power Amplifier; Hyper Rapid, LumeraLaser) which operates at a wavelength of $\lambda=355 \mathrm{~nm}$ with a pulse duration of $\tau<15 \mathrm{ps}$. The coherence length is $150 \mu \mathrm{m}$.

The coherence length describes the maximal possible offset in length of both beams relative to each other where beams are still partially coherent and interference can occur. An offset below the coherence length decreases the contrast of the interference pattern. While the other parameters which define the interference contrast as amplitude of each beam, polarization of each beam and quality of the superposition of beams can be easily measured and set to optimal values, the contribution of coherence of beams is difficult to quantify. A direct measurement of the interference pattern is usually not possible due to the resolution limit of sensors and the beams carrying no information afterwards. A measurement of the interference contrast is possible by a footprint method [14]. The measurement is based on a virtual shift of the ablation threshold due to redistribution of intensity by interference as shown in figure 6. As the modulation depth of the redistribution increases with the interference contrast also the shift of the measured ablation threshold increases to maximum of half of the real ablation threshold. To determine the ablation threshold a method by Liu is applied where a logarithmic fit is applied to graph where the radii of ablated areas over the peak fluence of a Gaussian beam are plotted [17].

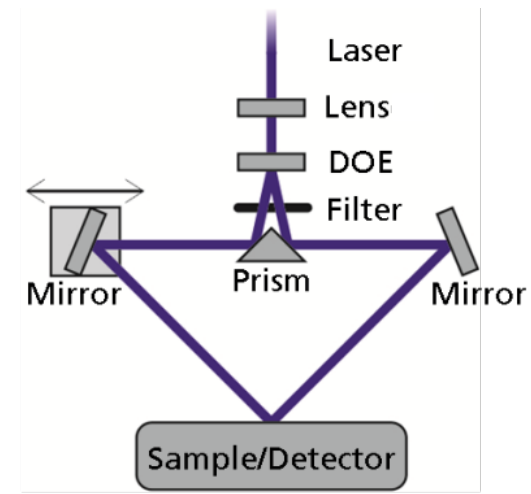

Fig. 5 The 2-beam interference setup with a diffractive optical element as beam splitter. The zero order and the higher orders than $1 / 1$ are blocked by filter. Each beam is deflected by a prism on a mirror. These mirrors guide the beams to the sample surface and their angle is adjustable to change the periodicity of the pattern. One mirror is also attached to a linear stage to allow an easy modification of the length of one beam path.

The direct surface structuring by MBI is tested on two materials: A $50 \mu \mathrm{m}$ thick polyimid film (Upilex) and polished stainless steel (1.4301) sheets. The output of both laser is measured by an energy detector (QE12LP-S-MP, Gentec) for the ns laser, by a power detector (UP19K-50L-W5, Gentec) for the ps laser and a beam profiler (Spiricon, Ophir) for both laser at the intersection of the beams. The diameter of the ablated area is measured by a Laser-Scanning Microscope (VK-9700, Keyence) and the geometry of generated structures is measured by Atomic-Force-Microscope (Rados N8, Bruker)

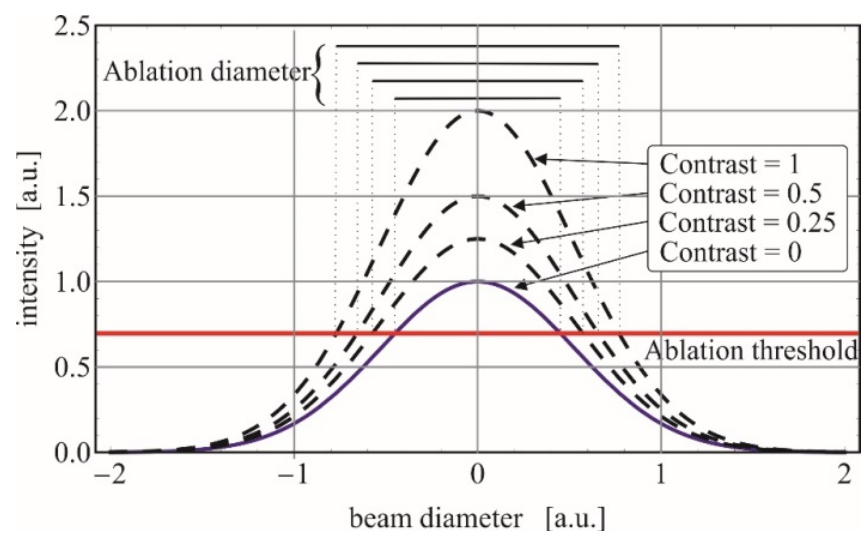

Fig. 6 Intensity profiles for the envelope of the peak intensities of the interference pattern. For a fixed total intensity $\boldsymbol{I}_{\mathbf{0}}$ the diameter of the area, where ablation occurs, increases in correlation with the interference contrast [15]. 


\section{Experimental results with ns-laser}

In the first experiment the ablation threshold on polyimide with and without interference is measured. For the ablation with interference all parameters are set to optimal values for an optimal interference contrast of 1: The energy of the beams is equal in intensity and distribution, the position of the sample surface is in the center of the interference volume, the polarization of both beams parallel and the length of the optical beam path set to a close value. The beam diameter at the sample surface is $740 \mu \mathrm{m}$ and is irradiated by one pulse. As shown in figure 7 a shift of the ablation threshold is observed: $124 \mathrm{~mJ} / \mathrm{cm}^{2}$ without interference and $72 \mathrm{~mJ} / \mathrm{cm}^{2}$ with interference. The interference contrast $\mathrm{K}$ can be calculated by:

$$
K=\frac{F_{\text {thres,without }}}{F_{\text {thres,interference }}}-1
$$

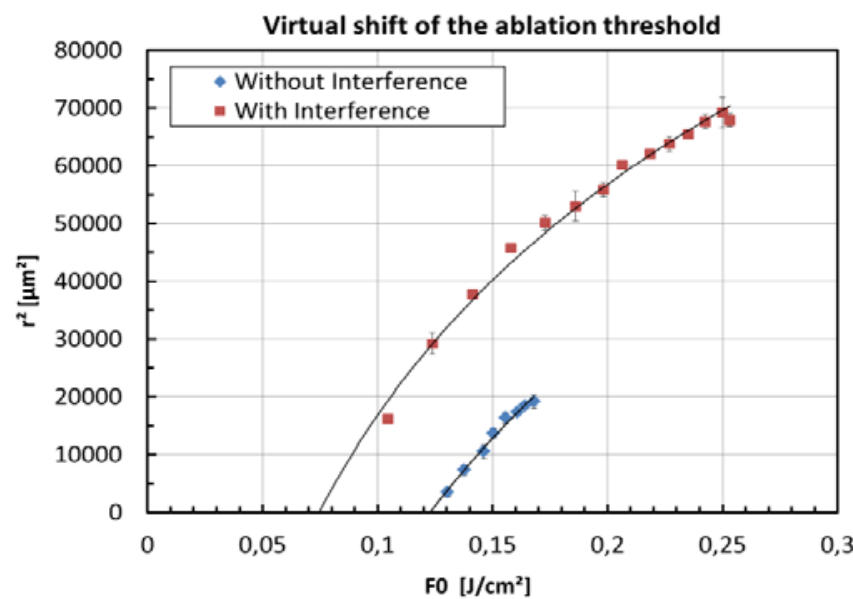

Fig. 7 Plot of the radii of the ablated spots against the peak fluence of the Gaussian laser beam. The intersection of logarithmic fit with $\mathrm{x}$-axis marks the ablations threshold for the polyimide foil with the ns-laser.

The calculated contrast is 0.71 which is lower than the expected optimal contrast of 1 . With the exception of the coherence of beams all relevant parameters for the contrast can be measured with high precision. The main reason for a reduced coherence is a deviation of the optical path length of the beams. In figure 8 the radii of the ablated areas over a shift of the optical path length of beams relative to each other is depicted while the total energy is kept constant. The measured results can be fitted by a Gaussian distribution with a width in good agreement with the coherence length of the laser. For larger shifts the radius stays constant and equals the ablation without interference.

After the method by Liu therefore the contrast decreases linear for a deviation in the optical path length of the beams. For shift larger than the coherence length the radii of spots are similar to ablation without interference. The peak of the Gaussian distribution fits well with the previously assumed equal path length of both beams. In figure 9 a set of microscope pictures of the ablated spots for different shift lengths is presented. Corresponding to the shift the optical impression of the spot changes. For higher contrast, which equals a low shift, color effects are visible presumably corresponding to the depth and width of the structures. These color effects reduce their intensity for lower contrasts and a ringlike behavior can appear. For translations larger than the coherence length the spot looks uniform and no color effects are visible.

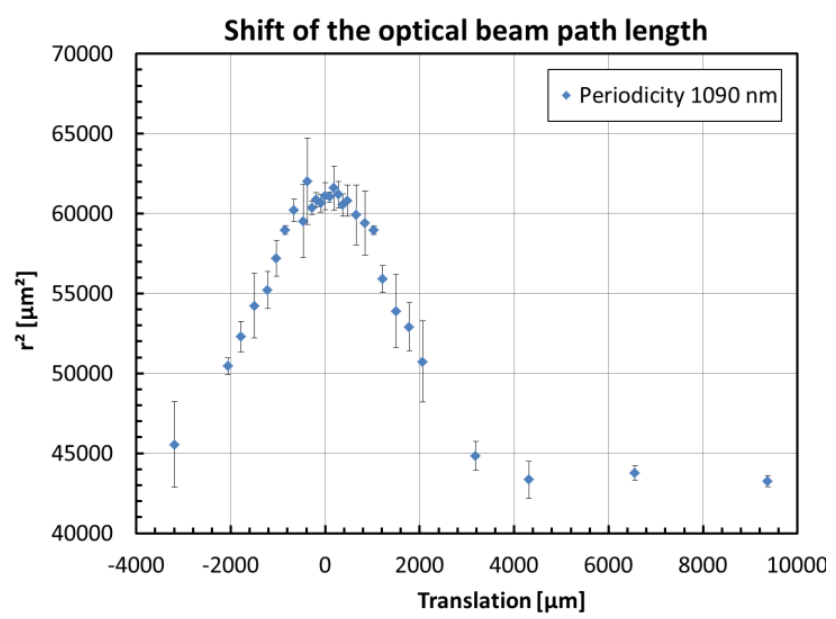

Fig. 8 Plot of the radii of generated spots against a translation of one beam relative to the other for the ns-laser source on polyimide. Between - $4 \mathrm{~mm}$ to $4.2 \mathrm{~mm}$ the curve can be fitted by Gaussian distribution. For larger shifts the radii are constant.

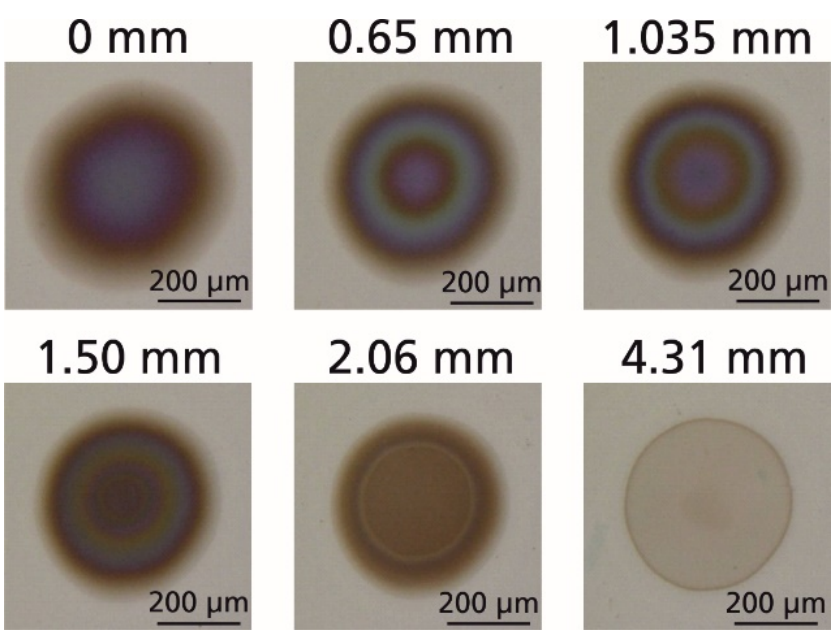

Fig. 9 Microscope pictures of the generated spots in polyimide for different shifts of the optical beam paths lengths relative to each other. For shifts lower than the coherence length ringlike color effects are visible which alter their shape depending on the size of the shift.

AFM measurements (figure 10) of the structures in the center of the spots show an increase of the width of structures while reducing the contrast as theoretically expected. For a shift of $1.8 \mathrm{~mm}$ the width equals the periodicity of the pattern. The depth first shows a slight increase before it declines as expected. This effect is probably caused by heating effects, e.g. melt expulsion which is not considered in the theoretical model. 


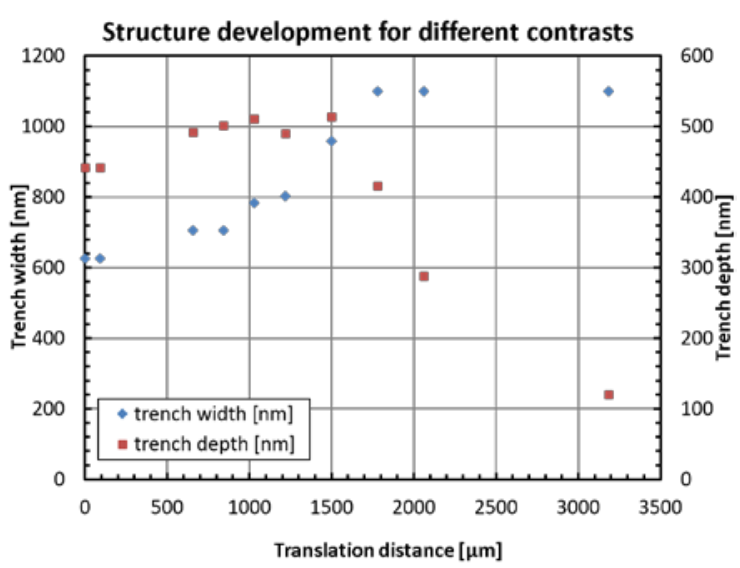

Fig. 10 Influence of translation and therefore reduced contrast on the depth and width of the generated structures in polyimide with the ns-laser. The periodicity of the interference pattern is $1100 \mathrm{~nm}$.

In last experiment the minimal periodicity of the pattern for direct surface structuring of polyimide with a ns-laser is tested. A minimal periodicity of $230 \mathrm{~nm}$ is reached with feature sizes in the $100 \mathrm{~nm}$ regime as visible in the extracted height profile, see figure 11 . Near the center of the AFM measurement first signs of deteriorations of the structures are visible, which increase for even smaller periodicities.
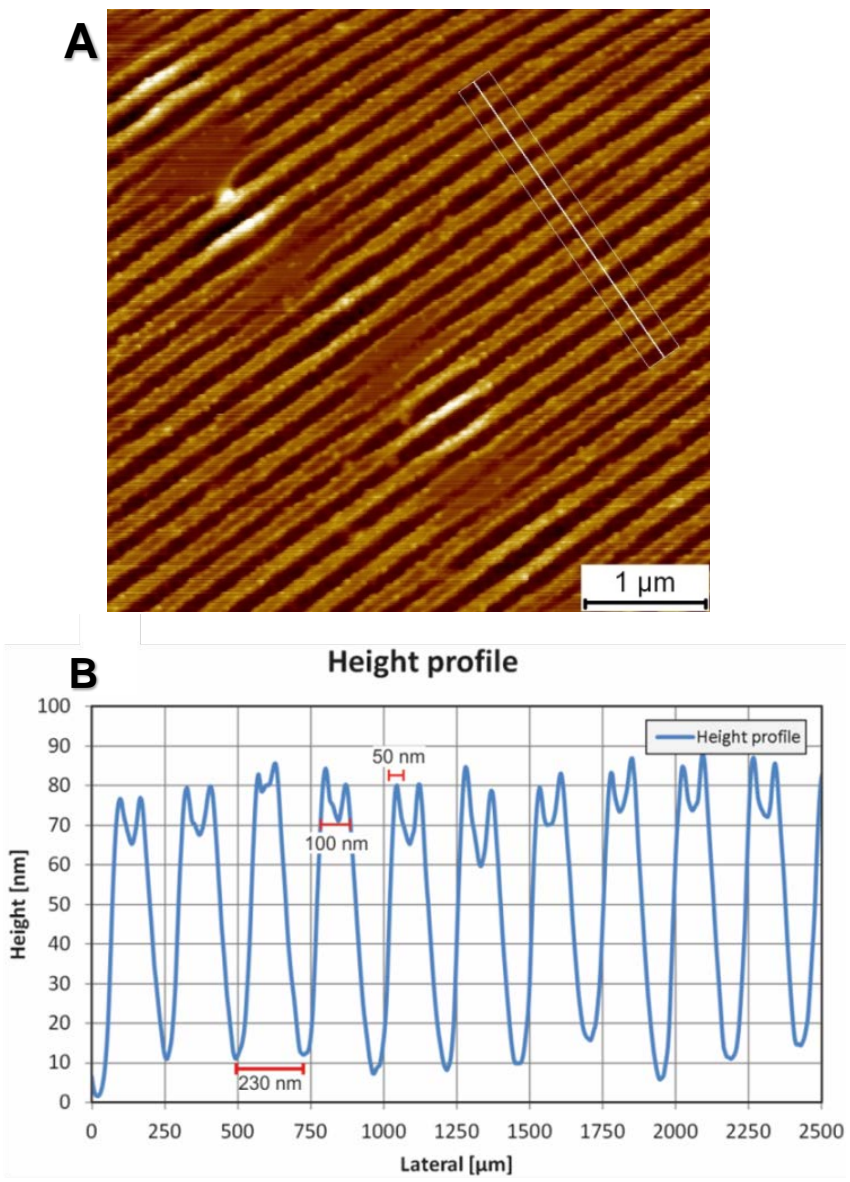

Fig. 11 (A) AFM measurement of structured polyimide with a periodicity of $230 \mathrm{~nm}$ created by a single pulse with a peak fluence of $98 \mathrm{~mJ}$. In the lower graph (B) an extracted height profile is shown.

\section{Experimental results with ps-laser}

In the first experiments the ablation or modification threshold on polyimide and stainless steel with and without interference is measured. For stainless steel these thresholds do not correspond necessarily to ablation, but can also equal an oxide based modification of the surface. The diameters of altered surfaces area are measured the LSM, which doesn't allow a distinction of these. The results of the measurements are shown in figure 12:

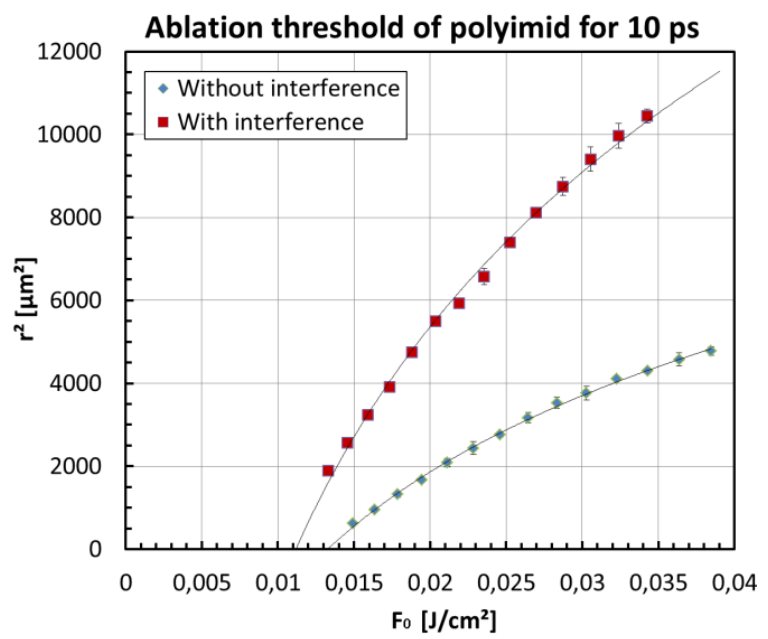

Fig. 12 Plot of the radii of the ablated spots against the peak fluence of the Gaussian laser beam. The intersection of the logarithmic fit with the x-axis marks the ablation threshold for polyimide with the ps-laser

For polyimide the ablation threshold without interference is $13.3 \mathrm{~mJ} / \mathrm{cm}^{2}$ and with interference $11.2 \mathrm{~mJ} / \mathrm{cm}^{2}$. The calculated interference contrast is 0.2 . For stainless steel the modification threshold without interference is $33 \mathrm{~mJ} / \mathrm{cm}^{2}$ and with interference $28 \mathrm{~mJ} / \mathrm{cm}^{2}$, which corresponds to an interference contrast of 0.18 , which is similar to the one for polyimide. Independent from the material, the interference contrast is significantly decreased for ultrashort pulses.

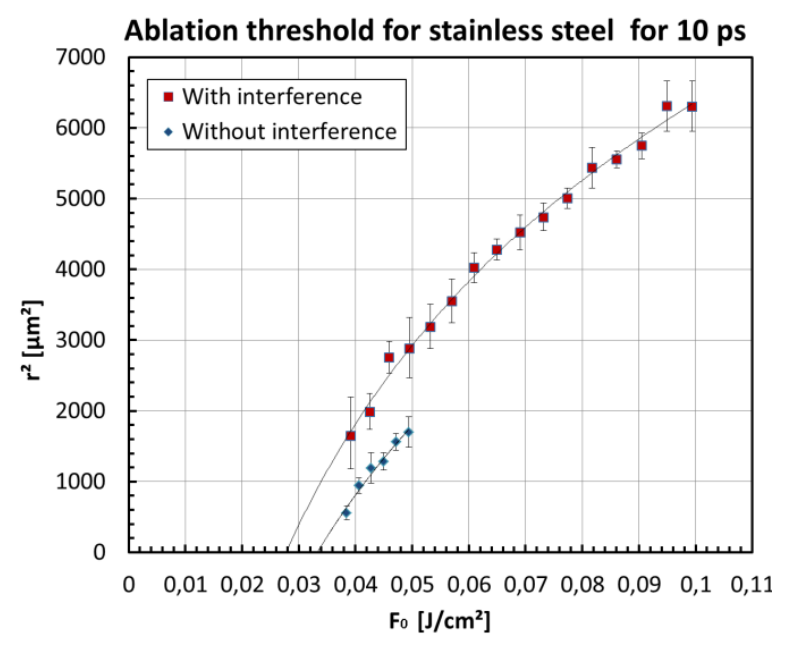

Fig. 13 Plot of the radii of the ablated spots against the peak fluence of the Gaussian laser beam. The intersection of the logarithmic fit with the x-axis marks the ablation threshold for stainless steel with the ps-laser. 
To verify that deviations in the optical path length of the beams are sufficiently small, the influence of a translation on radii of the spots is measured.

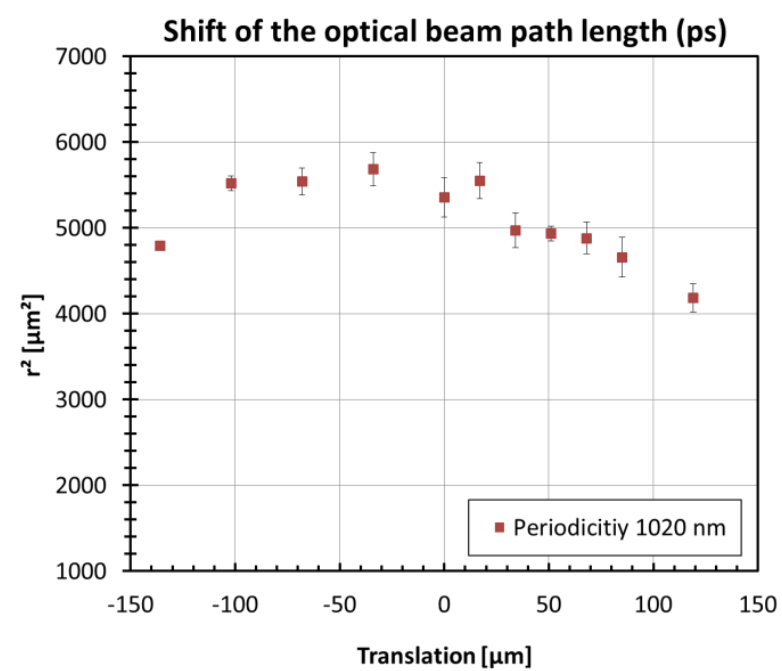

Fig. 14 Plot of the radii of generated spots against a translation of one beam relative to the other for the ps-laser source on stainless steel.

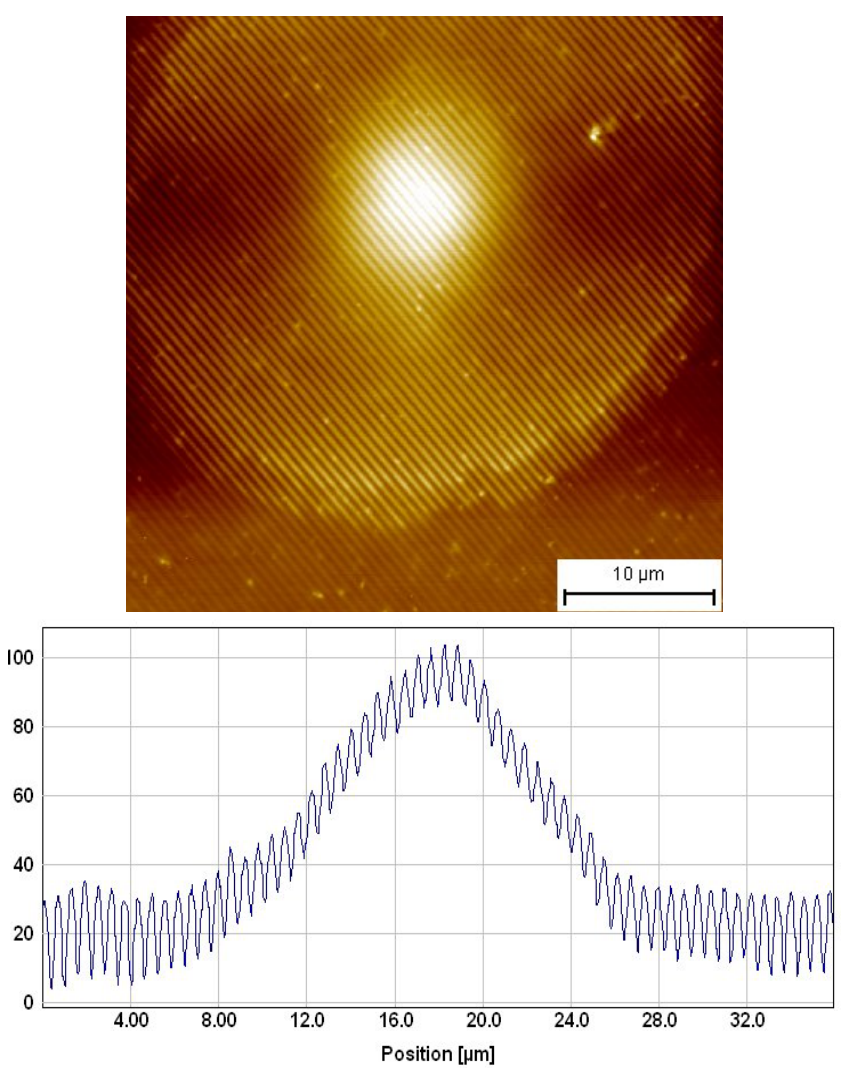

Fig. 15 AFM measurement of structured polyimide sample with a periodicity of $1020 \mathrm{~nm}$ by ps-laser. In the lower graph an extracted height profile is shown.

The measured values show a much larger variance in radii than for the ns experiments. The highest radius is observed for $-30 \mu \mathrm{m}$. The measurement of the ablation threshold was repeated for this optimized path length. The threshold is reduced to $25.5 \mathrm{~mJ} / \mathrm{cm}^{2}$ which improves the calculated interference contrast to 0.28 . Due to the shorter coherence length even small shifts in the optical path length of both beams have a strong influence on the contrast of the interference modulation. As the optical beam paths are difficult to adjust in the low $\mu$ m-regime, a measurement of radii of ablated spots against a shift is helpful to adjust the length of the beam path length relative to each other.

As shown in figure 15 structuring of polyimide with pslaser is suboptimal compared to the ns-laser results. The structures generated by interference are superposed by a strong bulging of the surface. The periodicity of the structure is $1020 \mathrm{~nm}$ and the depth of the structures is $20 \mathrm{~nm}$ which means their magnitude is smaller than for the ns ablation. The bulging is presumably caused by energy deposited in the intensity minima of the pattern. Due to the low contrast the minima have a relative high fluence which leads to significant heating of the surface.

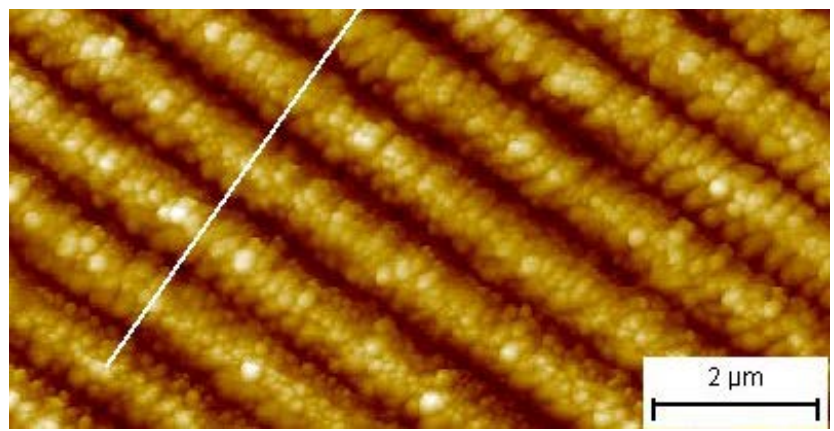

Fig. 16 AFM measurement of structured stainless steel sample with a periodicity of $1020 \mathrm{~nm}$ by ps-laser. The interference structure is superposed by ripples.
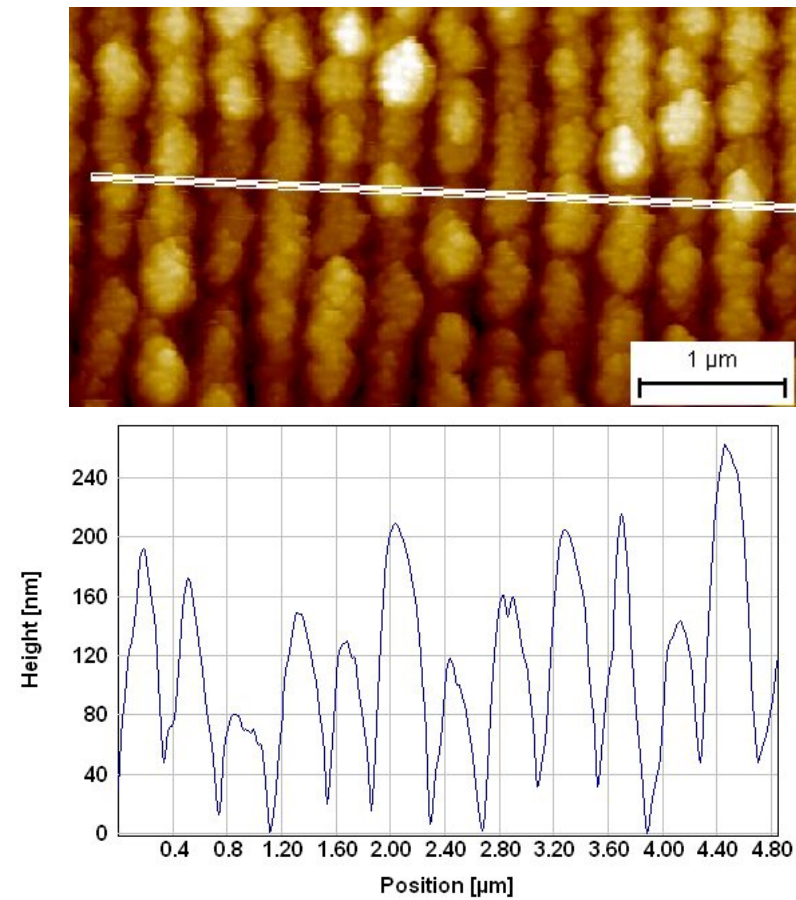

Fig. 17 AFM measurement of structured stainless steel sample with a periodicity of $1020 \mathrm{~nm}$ by ps-laser. The lower graph shows the height profile.

In the last experiment the structuring of stainless steel with interference and a ps-laser is tested. For a periodicity of $1020 \mathrm{~nm}$ grooves with a depth of $\sim 240 \mathrm{~nm}$ can be generated. Perpendicular to the interference structures ripples are induced by ultrashort pulse laser (figure 16). The periodicity 
of the ripples is in range of the laser wavelength. The minimal generated periodicity for stainless steel achieved is $400 \mathrm{~nm}$, at which point defects of the pattern are visible, that are presumably tied to the ripples. The depth of the structures show a strong variance in height and vary from 60 - $200 \mathrm{~nm}$.

\section{Conclusion}

For direct structuring of surfaces by MBI the periodicity and shape of the pattern, the phase stability and the interference contrast are important criteria. In respect to these criteria 2-Beam interference is the optimal setup for direct nano structuring of surfaces and its limitation on 1D structures can be partially circumvented by several consecutive structuring steps [4,12].

Although theoretically an ideal interference contrast of 1 is possible with 2-beam interference, experimental results indicate that the effective interference contrast is lower and tied to the coherence length of the laser. For the ns-laser the calculated contrast is 0.7 , while for the ps-laser the contrast is further reduced to 0.28 using the same experimental setup.

By means of the ns-laser minimal structure sizes near $100 \mathrm{~nm}$ can be generated in polyimide with a depth of $\sim 80 \mathrm{~nm}$. For ps-laser the structuring of polyimide is suboptimal compared to the ns-laser results. The depth of structures is significantly reduced and a strong bulging of the material occurs. Structuring of stainless steel with the interference pattern created by ps-laser is feasible. For a periodicity of $1020 \mathrm{~nm}$ well-defined structures can be generated with a depth of $\sim 240 \mathrm{~nm}$ which are superposed perpendicular by ripples structures with a periodicity of the laser wavelength. For a periodicity of $400 \mathrm{~nm}$ the depth of the structures vary significantly which is most likely due to the superposed ripples structures.

The coherence length of a laser is related to the pulse duration. Therefore the choice of the specific laser for structuring a surface by MBI interference depends on heat conductivity of the surface material and the structure size. The usage of ultrashort pulse laser for standard ablation conditions lead to relative melt-free structures due to the so called "cold ablation" process. The ultrashort pulses limit the heat diffusion in the material which is especially important for the generation of small features. For multi-beam interference this advantage is reduced or even inverted by the reduced interference contrast which is observed in this paper. Low contrast can lead to an energy deposition in the areas where no alteration of materials is wanted far greater than that caused by heat diffusion. For materials with low heat conductivity as plastics a ns-laser is more suitable, while for materials with high heat conductivity as metals a ps-laser is favorable.

\section{Acknowledgments and Appendixes}

Parts of the here depicted research was funded by the German Research Foundation (DFG) as part of the Cluster of Excellence "Integrative Production Technology for HighWage Countries" and as part of the DFG priority program "Generation of sub-100 nm structures by nonlinear lasermaterial interactions".

\section{References}

[1] S.H. Baik, S.K. Hwang, Y.G. Kim, G.J. Park, J.H. Kwon, W.T. Moon, S.H. Kim, B.K. Kim, and S.H. Kang: J. Opt. Soc. Korea, 13, (2009) 478.

[2] E. Bremus-Koebberling, S. Beckemper, B. Koch, and A. Gillner: J. Laser Appl., 24, (2012) 042013.

[3] D. Langheinrich, E. Yslas, M. Broglia, V. Rivarola, D. Acevedo, and A. Lasagni: J. Polym. Sci. Pol. Phys., 50, (2012) 415.

[4] M. Steger, C. Hartmann, S. Beckemper, S. Boes, J. Holtkamp, and A. Gillner: J. Laser Micro/Nanoengin., 8, (2014) 210.

[5] L.Z. Cai, X.L. Yang and Y.R. Wang: J. Mod. Opt., 49, (2002) 163.

[6] S. Beckemper, J. Huang, A. Gillner, and K. Wang: J. Laser Micro/Nanoengin., 6, (2011) 49.

[7] H.M. Phillips, D.L. Callahan, R. Sauerbrey, and G. Szabo. Z. Bor: Appl. Phys. Lett., 58, (1991) 2761.

[8] T. Tavera, N. Perez, A. Rodriguez, P. Yurrita, S.M. Olaizola, and E. Castano: Appl. Surf. Sci., 258, (2011) 1175.

[9] T. Lippert,T. Gerber, A. Wokaun, D. J. Funk, , H. Fukumura, and M. Goto: Appl. Phys. Lett. 75, (1999) 1018.

[10] R. Guo, Y. Dajun, and D. Suman: J. Micromech. Microeng., 21, (2010) 015010.

[11]A. Lasagni, F. Mücklich, M.R. Nejati, and R. Clasen: Adv. Eng. Mater., 8, (2006) 580.

[12] S. Indrisiunas, B. Voisiat, A. Zukauskas, G. Raciukaitis: Proc. SPIE Vol. 9350, (2015) 935003.

[13]B. Voisiat, M. Gedvilas, S. Indrisiunas, and G. Raciukaitis: J. Laser Micro/Nanoengin., 6, (2011) 185.

[14] G.M. Burrow, and T.K. Gaylord: J. Micro/Nanolith. MEMS MOEMS 11, 043004.

[15]E. Hecht: “Optik” (Oldenbourg, Munich, 2005) 624.

[16] M. Steger, S. Boes, S. Thilker, and A. Gillner: J. Laser Micro/Nanoengin., 9, (2013) 225.

[17] J.M. Liu: Opt. Lett., 7, (1982) 196.

(Received: May 26, 2015, Accepted: August 1, 2016) 